\title{
Green synthesis of pyrimidine derivative
}

\author{
Ratnamala P. Sonawane \\ The Institute of Science, Mumbai 400032, India \\ Email address: ratnasonawane@yahoo.in
}

\begin{abstract}
The majority of the drugs on the market today are entirely chemically synthesized in the laboratory. Several scientists had synthesized dihydropyrimidine (DHPMs) derivatives showing a wide spectrum of biological actions as antibacterials, antivirals as well as antitumor agents. This activity is principally due to presence of steriogenic carbon $\mathrm{C} 4$ in their structure. The current investigation is study and synthesis of dihydropyrimidinones efficiently and in high yields under mild, solvent free and eco-friendly conditions by using "Grindstone Chemistry Technique" catalyzed by $\mathrm{CuCl}_{2} \cdot 2 \mathrm{H}_{2} \mathrm{O}$ and Conc. $\mathrm{HCl}$. The obtained products have been identified by comparison with spectral data \& their melting points. The synthesis of DHPMs derivative involves a multicomponent reaction (aldehyde derivative, urea /thiourea and 1,3-dicarbonyl compounds) in presence of $\mathrm{HCl} /$ $\mathrm{NH}_{4} \mathrm{Cl}$ as a catalyst. 3,4-dihydropyrimidin-2-(1H)-ones was synthesized and characterized using IR. The melting points was obtained $203^{\circ} \mathrm{C}$.
\end{abstract}

Keyword: dihydropyrimidine derivatives; Biginelli Reaction; Grindstone chemistry

\section{INTRODUCTION}

The original one pot synthesis of 3,4-dihydropyrimidine-2-(1H)- ones was firstly reported by Pietro Biginelli in 1893 performing the three component cyclocondensation reaction of ethyl acetoacetate, benzaldehyde and urea under Bronsted acid catalysis ${ }^{1-2}$. However this reaction suffers from the harsh conditions, high reaction times and frequently low yields Aryl substituted 3,4-dihydropyrimidine-2-(1H)- ones ${ }^{3}$ and their derivatives are an important class of substances in organic and medicinal chemistry.

Many synthetic methods ${ }^{4-8}$ for preparing these compounds have been reported including classical methods, microwave irradiation and by using Lewis acids as well as protic acids. The discovery of a new an inexpensive catalyst for the preparation of 3,4-dihydropyrimidine$2-(1 \mathrm{H})$ - ones under neutral and mild conditions with high yield of prime importance. In solvent free organic reactions ${ }^{9}$ reagents react together in the absence of any solvent have been reviewed as a fast developing technology.

The development of new strategies for the synthesis of complex molecules is a challenging area of organic synthesis. A large number of organic reactions are typically carried out under anhydrous conditions, using volatile organic solvents like benzene which are the cause of environmental problems and also potentially carcinogenic. Hence, it is required to develope safe, practical and environmental friendly process. Many exothermic reactions can be accomplished in high yield by using a technique known as "Grindstone Chemistry" which is one of the "Green Chemistry Technique". 
Reactions are initiated by grinding, with transfer of very small amount of energy through friction. These new strategies involve catalysts such as $\mathrm{CeCl}_{3} \cdot 7 \mathrm{H}_{2} \mathrm{O}, \mathrm{FeCl}_{3}-\mathrm{HCl}$, $\mathrm{ZnCl}_{2}, \mathrm{CoCl}_{2}, \mathrm{CuCl}_{2} \cdot 2 \mathrm{H}_{2} \mathrm{O}$ etc.

Several marine alkaloids possessing the dihydropyrimidione moiety have shown interesting biological activities, for instance Batzelladine ${ }^{10}$ alkaloid has been reported to be a potent against HIV group. In recent years, development of more economical and environmentally friendly conversion processes is gaining interest in the chemical communities.

\section{EXPERIMENTAL}

The chemicals used Benzaldehyde, Ethyl acetoacetate, urea, thiourea, $\mathrm{CuCl}_{2} \cdot \mathrm{H}_{2} \mathrm{O}$, hydrochloric acid were of analytical reagent grade. A mixture of Benzaldehyde, ethylacetoacetate, urea, $\mathrm{CuCl}_{2} \cdot \mathrm{H}_{2} \mathrm{O}$ and few drops of $\mathrm{Conc}$. $\mathrm{HCl}$ was ground together for 2-5 min.

The solid mass was left overnight, then washed with cold water and purified by recrystallization from ethanol. The obtained products were identified from their spectral (IR) data and their melting point. Melting point was determined in open capillary tubes. The IR spectra were recorded on Shimadzu 435 FT- IR spectrophotometer with samples prepared as $\mathrm{KBr}$ palletes.

\section{1. Synthesis of 5-ethoxycarbonyl-4-phenyl-6-methyl-3,4-dihydropyrimidine-2-(1H)- one ${ }^{11}$ by conventional method}

A mixture of benzaldehyde $(1 \mathrm{mmol})$, ethyl acetoacetate $(1 \mathrm{mmol})$, urea/ thiourea (1mmol) and catalytic amount of $\mathrm{HCl} / \mathrm{NH}_{4} \mathrm{Cl}$ was taken in round bottom flask with $30 \mathrm{~cm}^{3}$ methanol and reflux at $100{ }^{\circ} \mathrm{C}$ for $3 \mathrm{hrs}$. The progress of the reaction was monitored by TLC. The mixture was cooled to room temperature and poured in cold water.

The solid product was collected by filtration. The product was dried and recrystllised from hot alcohol to obtained the pure product. Product was characterized by melting point, IR.

\section{2. Synthesis of 5-ethoxycarbonyl-4-phenyl-6-methyl-3,4-dihydropyrimidine-2-(1H)- one by Grindstone Chemistry}

A mixture of benzaldehyde $(1 \mathrm{mmol})$, ethyl acetoacetate $(1 \mathrm{mmol})$, urea/ thiourea (1mmol) and catalytic amount of $\mathrm{CuCl}_{2} \cdot \mathrm{H}_{2} \mathrm{O}$ was ground together for 2-5 min.

The solid mass was left overnight, then washed with cold water and the product was dried and recrystllised from hot alcohol to obtained the pure product.

The progress of the reaction was monitored by TLC Product was characterized by melting point, IR. 


\section{REACTION}
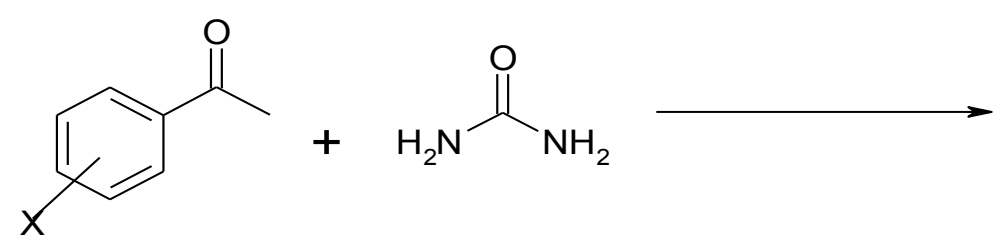<smiles>[X]c1cccc(C(O)NC(N)=O)c1</smiles><smiles>[X]c1cccc(C(C(C(C)=O)C(=O)OCC)N([2H])C(N)=O)c1</smiles><smiles></smiles>

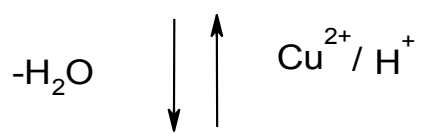<smiles>[14CH3]/C(=C/c1ccccc1)N([14CH3])C(N)=O</smiles><smiles>[X]c1cccc(C2C(C(=O)OCC)N(C)C(=O)NC2(C)O)c1</smiles><smiles>[X]c1cccc(C2C(C(=O)OCC)=C(C)NC(=O)N2C)c1</smiles>

$\mathrm{x}=\mathrm{H} / \mathrm{Cl} / \mathrm{NO}_{2} / \mathrm{OMe}$ 


\section{RESULTS AND DISCUSSION}

Table 1. Interpretation of IR spectra of 5-ethoxycarbonyl-4-phenyl-6-methyl-3,4-dihydropyrimidine$2-(1 \mathrm{H})$-one by conventional method.

\begin{tabular}{|c|c|c|}
\hline IR range $\left(\mathbf{c m}^{-\mathbf{1}}\right)$ & Peak Intensity & Peak due to \\
\hline 3247.99 & Sharp & N-H Stretching \\
\hline 3116.80 & Sharp & Aromatic C-H Stretching \\
\hline 2980.56 & Sharp & Aliphatic C-H Stretching \\
\hline 1725.85 & Sharp & Ester \\
\hline 1702.16 & Sharp & C=C Stretching \\
\hline 1650.57 & Sharp & Asymmetric C-H bending \\
\hline 1465.18 & Sharp & C-O Stretching \\
\hline 1270.88 & Sharp & Mono substituted benzene \\
\hline 700.46 & Sharp & \\
\hline
\end{tabular}

Table 2. Interpretation of IR spectra of 5-ethoxycarbonyl-4-phenyl-6-methyl-3,4-dihydropyrimidine2-(1H)-one by Grindstone Chemistry.

\begin{tabular}{|c|c|c|}
\hline IR range $\mathbf{( c m}^{-\mathbf{1}} \mathbf{)}$ & Peak Intensity & Peak due to \\
\hline 3288.74 & Sharp & N-H Stretching \\
\hline 2955.04 & Sharp & Aromatic C-H Stretching \\
\hline 2980.56 & Sharp & Aliphatic C-H Stretching \\
\hline 1720.35 & Sharp & Ester \\
\hline 1726.35 & Sharp & C=C Stretching \\
\hline 1635.69 & Sharp & Asymmetric C-H bending \\
\hline 1455.20 & Sharp & C-O Stretching \\
\hline 1290.42 & Sharp & Mono substituted benzene \\
\hline 698.2 & Sharp & \\
\hline
\end{tabular}




\section{CONCLUSION}

The preparation of 5-ethoxycarbonyl-4-phenyl-6-methyl-3,4-dihydropyrimidine-2$(1 \mathrm{H})$-one by Grindstone Chemistry shows synthesis is in good yields and in less time and also avoids problems associated with solvent use. It was found that $\mathrm{CuCl}_{2} \cdot \mathrm{H}_{2} \mathrm{O}$ works as an excellent catalyst for the one-pot three components and solvent free synthesis of dihydropyrimidione.

This technique is superior to the existing methods. Since grinding does not require solvents leading to a safe and environmental friendly synthesis solvent free approach opens up numerous possibilities for conducting rapid organic synthesis and functional group transformations more efficiently. Additionally there are distinct advantages of these solvent free reactions. It prevents pollution in organic synthesis at source.

\section{References}

[1] P. Biginelli, Chem Ber 24 (1891) 1317.

[2] F. Makaev, E. Styngach, V. Muntyanu, S.Pogrebnoi, Russian Journal of Organic Chemistry 43 (2007) 1512-1515.

[3] A. Shaabani, A. Bazgir, F. Teimouri, Tetrahedron Letters 44(4) (2003) 857-859.

[4] S. V. Ryabukhin, A. S. Plaskon, E. N. Ostapchuk, D. M. Volochnyuk., Synthesis 3 (2007) 417-427.

[5] I. Cepanec, M. Litvic, A. Bartolincic, Tetrahedron 61 (2005) 4275-4280.

[6] J. H. Schauble, E. A. Trauffer, P. P. Deshpande, R. D. Evans, Synthesis (2005) 1333-1339.

[7] H. Hazarkhani, B. Karimi, Synthesis (2004) 1239-1242.

[8] J. Lu, Y. Bai, Synthesis (2002) 466-470.

[9] Tom Welton, Green Chem 8 (2006) 13.

[10] Hue-Ming Hua, Jiangnan Peng, Tetrahedran 63(45) (2007) 11179-11188.

[11] R. P. Sonawane, International Letters of Chemistry, Physics and Astronomy 5 (2013) 7-11. 